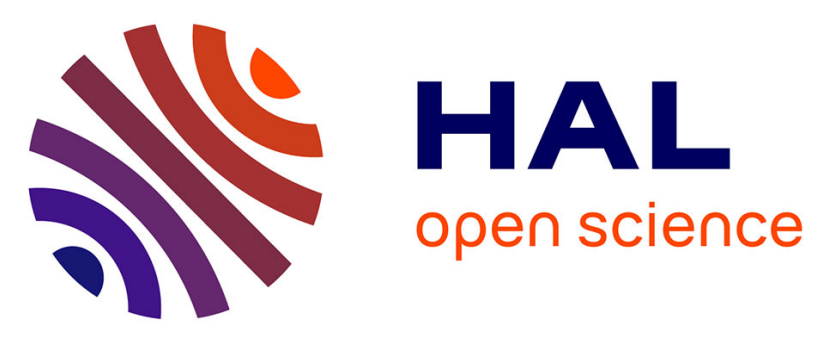

\title{
Les internationales ouvrières
}

Samuel Hayat

\section{To cite this version:}

Samuel Hayat. Les internationales ouvrières. Le Magasin du XIXe siècle, 2018, Magasin du XIXe siècle, 8, pp.126-131. hal-02367988

\section{HAL Id: hal-02367988 \\ https://hal.univ-lille.fr/hal-02367988}

Submitted on 18 Nov 2019

HAL is a multi-disciplinary open access archive for the deposit and dissemination of scientific research documents, whether they are published or not. The documents may come from teaching and research institutions in France or abroad, or from public or private research centers.
L'archive ouverte pluridisciplinaire HAL, est destinée au dépôt et à la diffusion de documents scientifiques de niveau recherche, publiés ou non, émanant des établissements d'enseignement et de recherche français ou étrangers, des laboratoires publics ou privés. 


\section{Les internationales ouvrières}

Publié dans Magasin du XIXe siècle, $\mathrm{n}^{\circ}$ 9, « Cosmopolis », p. 126-131

L'histoire des internationales ouvrières s'inscrit dans le large mouvement d'internationalisation que connaît l'Europe au XIXe siècle. L'expérience du Cosmopolis, de l'unification du monde comme monde, fût-elle très située et largement imaginaire, est alors concurrencée par l'émergence d'un ordre international, c'est-à-dire d'un monde comme ensemble d'Etats-nations dont les frontières se précisent et se renforcent, mais peuvent parfois être franchies. Il ne faudrait pas trop durcir l'opposition, car le langage ne cesse de glisser au gré des besoins, mais la distinction analytique vaut: l'international, contrairement au cosmopolite, suppose un déploiement matériel, il n'existe qu'incarné dans des dispositifs internationaux qui traversent explicitement les frontières nationales, sans véritablement les mettre en question: sommets de chefs d'Etat, compagnies commerciales, associations internationales. Il serait heureux de pouvoir séparer a priori ce qui, dans le mouvement d'internationalisation de l'Europe, engage l'humanité dans l'horreur - la colonisation, la guerre, l'exploitation - et ce qui l'élève - la solidarité internationale, les échanges dans les sciences et les arts. Mais ces mouvements ne cessent d'aller de pair : l'internationalisation des insurrections et celle de leur répression, l'internationale libérale et la Sainte-Alliance de Metternich, la mondialisation capitaliste et la résistance ouvrière internationale.

L'internationalisme ouvrier s'insère dans cette dynamique contradictoire, dès ses premières expressions. S'il y avait des contacts entre milieux ouvriers des deux côtés de la Manche bien avant cette date, c'est à l'occasion de l'Exposition universelle de Londres, en 1862, que le projet d'une association internationale ouvrière s'ébauche. Des rencontres ont alors lieu entre la délégation d'ouvriers français envoyée par Napoléon III et des syndicalistes anglais, dans les interstices de cet événement tout à la gloire de la machine et du capitalisme. L'année suivante, certains délégués français, dont le bronzier Henri Tolain, retournent à Londres pour un rassemblement international contre la répression tsariste de l'insurrection polonaise. Capitalisme et répression : l'internationalisme ouvrier se développe et se déploie parallèlement aux dynamiques d'oppression auxquelles il s'oppose.

Le 4 novembre 1864, un nouveau meeting londonien, à Saint-Martin's Hall, aboutit à la création de l'Association internationale des travailleurs (AIT), bientôt simplement appelée l'Internationale. Ce raccourci, de l'AIT à l'Internationale, n'est pas une construction d'historien, c'est bien ainsi que ses partisans comme ses adversaires désignent l'AIT dès ses premières années. Mais l'Internationale n'est pas seulement l'adjectif féminin substantivé de l'Association internationale des travailleurs, ni le bel hymne écrit en 1871 par Eugène Pottier et mis en musique en 1888 par le Lillois Pierre Degeyter, ni l'espoir d'une République vraie l'Internationale, qui « sera le genre humain », comme la Sociale. Hasard des noms ou signe de l'époque, l'AIT n'est pas la seule Internationale à apparaître en 1864 : quelques mois avant sa fondation, un décret impérial avait autorisé la création de "l'Internationale, compagnie d'assurances maritimes et fluviales », réunissant 72 actionnaires, propriétaires, banquiers, proches de Napoléon III, l'alliance entre les despotismes financier et impérial. Deux conceptions de l'internationalisation se donnent à voir dans cette symétrie : Internationale ouvrière contre Internationale capitaliste, les Etats pesant de tout leur poids pour que la seconde triomphe de la première. Et on le sait, le long XIXe siècle s'achèvera dans la boue des tranchées, le triomphe de l'industrie militaire et l'amoncellement de cadavres, sans que l'Internationale ouvrière - la Deuxième Internationale, fondée en 1889 - ne puisse rien 
empêcher, quoi qu'elle en ait et malgré les 3,5 millions d'adhérents des partis socialistes qu'elle regroupait alors.

C'est à l'horizon de cette catastrophe qu'il faut lire l'histoire des internationales ouvrières, car celles-ci ne cessent de se donner pour but explicite de mettre en échec la politique tyrannique des gouvernements européens et l'internationalisation du capitalisme. C'est ce qui ressort de l'adresse de 1864, écrite l'année précédente, que lit le leader tradeunioniste George Odger, « au nom des ouvriers d'Angleterre », aux ouvriers français à SaintMartin's Hall. D'un côté, dit-il, « les rois et les empereurs ont leurs réunions et leurs festins » où «les crimes réussis se voient justifiés », et face à cela il faut « une union fraternelle des peuples comme un moyen de mettre un terme aux abus actuels du pouvoir [...] despotique [des] fourbes roublards »- Odger cite les interventions impériales en Italie, au Mexique, en Suisse, en Chine. L'internationalisme est ici une forme de fraternité opposée au despotisme impérialiste. D'un autre côté, c'est la question sociale qui se joue dans l'internationalisation, car «toutes les fois que nous essayons d'améliorer notre condition sociale en réduisant les heures de labeur, ou en augmentant le prix du travail, nos patrons nous menacent d'amener des Français, des Allemands, des Belges et d'autres pour faire notre travail en acceptant des salaires inférieurs ». Face à cette mise en concurrence, qui est le sens même de l'internationalisation capitaliste, il faut «une union fraternelle entre les peuples», des « rapports réguliers et systématiques entre les classes ouvrières de tous les pays » afin de «ne pas permettre à nos patrons de créer la discorde entre nous et de nous réduire à la condition la plus misérable, au gré de leurs cupides marchandages ». Il se dégage de l'adresse d'Odger la perception d'une continuité entre exploitation capitaliste, domination despotique de l'Etat et maintien sous tutelle des masses de travailleurs ; il se lit aussi la croyance dans la possibilité de mettre fin à cet engrenage mortifère par la solidarité internationale.

La réponse des délégués ouvriers français, lue par Tolain, suit la même partition: despotisme des tyrans - «Il ne faut plus que des Césars, le front souillé d'une couronne sanglante, se partagent entre eux des peuples épuisés par les rapines des grands, des pays dévastés par des guerres sauvages »; despotisme du capital - «Progrès industriel, division du travail, libre échange [...], les capitaux se concentrent, s'organisent en puissantes associations financières et industrielles. Si nous n'y prenons garde, cette force sans contrepoids régnera bientôt despotiquement » faisant de «chaque ouvrier un rouage dans la main des hauts barons de l'industrie ». Et face à cette menace, une seule possibilité, se rencontrer, se reconnaître, lutter ensemble : «Donc, ceignons nos reins, préparons-nous avec joie à la lutte, Il faut que le peuple fasse entendre sa voix dans toutes les grandes questions politiques et sociales, signifiant ainsi aux despotes que la fin de leur tyrannique tutelle est arrivée. Travailleurs de tout pays qui voulez être libres : à votre tour d'avoir des congrès. C'est le peuple qui revient enfin sur la scène, ayant conscience de sa force et se dressant en face de la tyrannie dans l'ordre politique, en face du monopole, du privilège dans l'ordre économique. » Et il conclut : «Sauvons-nous par la solidarité ! »

S'agit-il là d'une injonction moralisatrice, de cet humanisme que Marx détestait tant chez ceux qu'il appelait «ces ânes de proudhoniens », les membres de la première section parisienne de l'AIT, sise rue des Gravilliers ? Ce serait oublier que derrières ces mots, des mécanismes de solidarité concrets se mettent en place. L'internationalisme n'est pas seulement une analyse des liens entre le capitalisme et la tyrannie, encore moins un simple état d'esprit fraternitaire, ce sont des échanges d'information, des soutiens financiers aux grèves, un modèle organisationnel auquel les travailleurs de tous les pays peuvent s'accrocher pour développer des sections locales - ce qui se fait surtout en Europe mais aussi ailleurs, notamment aux Etats-Unis. C'est cette ambition pratique qui rend l'Internationale efficace et donc dangereuse pour l'Etat et le capital. 
A la fin des années 1860, une vague de grèves secoue l'Europe, notamment en France, en Belgique, en Suisse, où les ouvriers demandent entre autres la journée de dix heures. Dans ces pays, l'Internationale se développe fortement, ce qui convainc les gouvernements que derrières les grèves se trouvent la main de l'étranger - alors que bien sûr, comme l'écrit le Conseil Général dans son rapport au Congrès de Bâle de septembre 1869 à propos de la grève des ovalistes, à Lyon, ces ouvrières qui avaient demandé à l'Internationale un soutien dans leur grève, «ce n'était pas l'Internationale qui jeta les ouvriers dans la grève, mais la grève qui les jeta dans l'Internationale ». En France, les procès se multiplient. La décision de l'AIT de soutenir la Commune de 1871, malgré l'opposition d'une partie de ses membres, en particulier les trade-unionistes anglais, est un tournant. Elle révèle que l'internationalisme ouvrier n'est pas une vaine invocation de principes généreux, mais un outil de lutte qui vise la transformation révolutionnaire, socialiste et ouvrière de l'Europe. Après l'insurrection, la courte expérience de la liberté puis les massacres du printemps 1871, l'Internationale devient l'ennemi à abattre, le nom même du mal qui pousse les ouvriers à sortir du rang et défendre leurs droits. Une campagne est lancée, commençant avec une enquête parlementaire sur l'insurrection du 18 mars 1871, se poursuivant avec nombre de livres d'auteurs libéraux ou conservateurs : Charles de Mazade, Félix Lequien, Edmond Villetard, Oscar Testut, Claudio Jannet, Pierre Zaconne. Qu'ils soient tricolores, bleus ou blancs, ces bons esprits puisent indistinctement dans la grande tradition réactionnaire de la dénonciation des complots maçonniques, jacobins, libéraux, socialistes qui a toujours accompagné les mouvements d'émancipation. Après la fusillade et la calomnie, la campagne contre l'internationalisme ouvrier en France prend l'apparence de la légalité avec la loi Dufaure du 14 mars 1872 qui punit de prison l'appartenance ou le soutien à «toute association internationale qui, sous quelque dénomination que ce soit et notamment sous celle d'association internationale des travailleurs, aura pour but de provoquer à la suspension du travail, à l'abolition du droit de propriété, de la famille, de la patrie ou des cultes reconnus par l'Etat ».

Ce n'est pourtant pas la répression qui met fin à l'expérience de la Première Internationale, et qui condamne la Deuxième - cette numérotation n'apparaît qu'a posteriori, en 1914 - à l'impuissance face à la guerre. Le Congrès de La Haye, en septembre 1872, voit l'exclusion des antiautoritaires, qui se rassemblent à Saint-Imier quelques jours plus tard, actant de fait la division du mouvement ouvrier international. Les raisons de la division sont connues : conflit de personnes (notamment entre Marx et Bakounine), divergences de vues sur le rôle du pouvoir d'Etat (à saisir ou à détruire) et surtout sur le fonctionnement de l'organisation elle-même (centralisée ou reposant sur l'autonomie des sections). Il se dessine là deux manières inconciliables de faire internationale, toutes deux traversées de contradictions. Les antiautoritaires veulent un internationalisme décentralisé, respectueux de l'autonomie des mouvements ouvriers nationaux, préfiguratif, comme le formulent les délégués à Saint-Imier, "d'une organisation et d'une fédération économiques absolument libres, fondées sur le travail et l'égalité de tous et absolument indépendantes de tout gouvernement politique », amenées à remplacer les Etats. Mais comment construire un sujet politique international, seul adapté à l'internationalisation du capitalisme, capable d'obtenir «la destruction de tous les États nationaux » souhaitée par Bakounine à Bâle en 1869, si l'Internationale se borne à être un simple «pacte d'amitié, de solidarité et de défense mutuelle»? Malgré les efforts de la Fédération jurassienne dans les années 1870, les mouvements inspirés par l'internationalisme antiautoritaire, en particulier anarchosyndicalistes, se développent de manière largement indépendante.

Du côté du Conseil général, dominé par Marx, faire internationale suppose une action coordonnée, centralisée, dirigée par Londres, quitte à faire fi de la volonté des mouvements ouvriers de chaque pays. C'est là se donner les moyens de constituer un réel appareil de 
représentation de la classe ouvrière internationale, mais s'engager dans la voie politique amène à se limiter à la scène de l'Etat-nation - ce qui amène Friedrich Engels à se montrer réticent à la reconstruction d'une Internationale en l'absence de partis socialistes nationaux puissants. Comme l'écrit en 1905 le socialiste roumain Christian Rakovsky, "même le socialisme le plus internationaliste procède par des voies nationales dans la mesure où chaque Parti socialiste est organisé de manière nationale avant de l'être de manière internationale. » Cette tension n'est jamais résolue, pas plus dans l'AIT débarrassée des anarchistes, qui survit jusqu'en 1876, que dans la Deuxième Internationale, fondée en 1889, cette fois sous une direction exclusivement marxiste, actée par le congrès de Londres de 1896 qui affirme le primat de l'action législative et parlementaire. Les socialistes connaissent bien des succès électoraux et intègrent progressivement l'élite étatique de leur pays, mais cela ne s'accompagne pas d'un développement de l'internationalisme. Au contraire, malgré l'institution en 1900 d'un Bureau Socialiste International, bientôt dirigé par le Belge Camille Huysmans, la Deuxième Internationale ne devient jamais rien de plus qu'un outil de collaboration, de discussion, parfois de solidarité, entre partis socialistes nationaux, sous la domination des plus importants d'entre eux, le français et surtout l'allemand. A l'intérieur de ces partis nationaux, de véritables débats ont lieu, notamment sur la question des nationalités au sein du prolétariat du pays - en particulier dans les Etats multinationaux comme la Russie et l'Autriche, là où existent des partis socialistes juifs ou encore pour les peuples répartis entre plusieurs Etats, comme les Polonais. Mais ces débats ne se trouvent jamais réellement pris en charge par l'Internationale, sauf de manière ponctuelle.

Rien ne dit mieux l'échec de l'internationalisme socialiste que le rapport à la colonisation, cette entreprise d'exploitation et de destruction dans laquelle s'enfoncent alors les pays européens. Certes, quand la question est soulevée dans les congrès de l'Internationale, ce qui est rare, le colonialisme capitaliste est dénoncé, mais chez beaucoup c'est au profit d'une politique coloniale dite positive qui «en régime socialiste pourra être une œuvre de civilisation », selon les termes de la motion défendue par le Néerlandais Henri Van Kol au Congrès de Stuttgart en 1907, qui n'échoue que du fait de l'intervention vigoureuse de Karl Kautsky. La détérioration de la situation dans les Balkans, les révolutions en Turquie en 1908, en Iran et en Chine en 1911, créent un regain d'intérêt pour les questions internationales chez les socialistes, mais achèvent de faire passer le problème colonial au second plan. L'adoption des règles du jeu de la politique étatique laisse peu de place pour un internationalisme qui serait un véritable dépassement des logiques impérialistes et guerrières de l'intérêt national.

De là, peut-être, vient l'impuissance de l'Internationale face à l'entrée en guerre des Etats européens. Ce ne sont pas les principes qui manquent, pourtant. Réunis à Bâle en novembre 1912, les socialistes déclarent « guerre à la guerre » et veulent opposer « au monde capitaliste de l'exploitation et du meurtre les masses du monde prolétarien de la paix et de l'Union des peuples ». Mais face à l'emballement de la mécanique guerrière, l'Internationale se révèle vide de toute volonté collective. Le 29 juillet 1914, le Bureau socialiste international se réunit à Bruxelles et appelle à un congrès en urgence à Paris le 9 août. Le congrès n'aura jamais lieu, car la guerre éclate entre temps, et tous les partis socialistes européens votent les crédits de guerre et collaborent avec les gouvernements bourgeois, excepté en Russie et en Serbie. L'Internationale ouvrière s'y révèle comme n'ayant jamais été plus que l'agrégation de partis nationaux, fussent-ils socialistes, saisis au moment de la guerre par des logiques internationales qui les rabattent vers le patriotisme.

Dans un texte d'avril 1916, alors que la guerre consume les peuples, Rosa Luxembourg écrit : «Le 4 août 1914, la social-démocratie allemande officielle et, avec elle, l'Internationale, s'effondrent misérablement. Tout ce que, pendant les cinquante années 
précédentes, nous avions prêché au peuple, que nous avions déclaré être nos principes sacrés [...] se révéla être un vide boniment. Soudainement, comme par magie noire, le parti de la lutte de classe internationale prolétarienne est devenu un parti libéral national. [...] Nous sommes devenus les outils indécis et justement méprisés de notre ennemi mortel, la bourgeoisie impérialiste. [...] Le vieux cri orgueilleux : «Prolétaires de tous les pays, unissezvous !» a été transformé sur le champ de bataille en un ordre : "Prolétaires de tous les pays, égorgez-vous ! » " L'internationalisme ouvrier, tel qu'il avait pu être rêvé et pratiqué au XIXe siècle, ne se relèvera pas de l'épreuve et laissera place, après la guerre, à une manière de faire internationale sous la seule direction autoritaire de l'Union soviétique.

\section{Samuel Hayat, CNRS (CERAPS)}

Dogliani Patrizia, «The Fate of Socialist Internationalism », in Glenda Sluga et Patricia Clavin (dirs.), Internationalisms. A Twentieth-Century History, Cambridge, Cambridge University Press, 2016, pp. 38-60.

Haupt Georges et Rebérioux Madeleine (dir.), La Deuxième Internationale et l'Orient, Paris, Editions Cujas, 1967.

Les Internationales et le problème de la guerre au XXe siècle, Ecole française de Rome, 1987.

Weill Claudie, «Les Internationales et la question nationale », in Jean-Jacques Becker et Gilles Candar(dirs.), Histoire des gauches en France, Paris, La Découverte, coll. « Poche/Sciences humaines et sociales », 2005, pp. 489-505. 